\title{
Review of: "Adolescent ethanol drinking promotes hyperalgesia, neuroinflammation and serotonergic deficits in mice that persist into adulthood"
}

\author{
Giselle Prunell
}

Potential competing interests: The author(s) declared that no potential competing interests exist.

In this manuscript, Khan et. al. report that adolescent intermittent ethanol consumption in mice results in long lasting brain alterations: adult mice that were exposed to ethanol during adolescence exhibit increased pain sensitivity and depressive-like behaviors, associated to microglia activation in the raphe nuclei and alterations in the serotonergic system. Minocycline treatment, that inhibits microglia activation, prevents ethanol-induced hyperalgesia and social interaction deficits. Besides, chemogenetic activation of microglia in the dorsal raphe nucleus was sufficient to induce hyperalgesia and social deficits in control mice. These results strongly suggest that ethanol consumption in the adolescence mice results in persistent microglia activation in the dorsal raphe nucleus, affecting the serotonergic system and leading to pain and affective disorders in the adult life.

This manuscript is very interesting with solid data to support the main conclusions and contributes to the knowledge of the field. However, the introduction and discussion are focused on the effect of ethanol adolescence consumption and nociception, while the studies about social interaction/depressive like behavior are not well supported and not discussed at all. These results are very interesting too, and it could be valuable to better explain the reasons to include these studies and discuss the results obtained.

Below find some comments and suggestions that might improve the manuscript:

In the Introduction secction: the actual knowledge about the relationship of adolescence etanol intakeaffective disorders- serotonergic system-neuroinflammation should be presented.

In the Methods section, some procedures and methodologies are poorly explained. Below are some examples:

-The age of the animas used for the ethanol treatment experiments and for the DREADD experiments should be mentioned.

-The information provided in the in the Supplementary Material, Intermittent access to ethanol is relevant to understand the experimental design. Thus, I recommend to include it in the main part of the manuscript.

- Minocycline treatment should be better described (was it co-administered with ethanol, was it administered after the ethanol treatment, for how long, ...)

- The section 2.4. Stereotaxic surgeries could be included in section 2.5.4. DREADD experiments, since 
both are related to the same experiment.

- The procedure used for control/sham animals in the DREADD experiments should be described.

In the Results section:

- Some of the statistics mentioned needs better description. For example: Von Frey: $t 17=4.11, p<0.001$, it is not indicated to which filament this analysis corresponds; Hargreaves t18 $=3.05, p<0.01$, does this value corresponds to threshold or latency?

- Depression and anxiety are strongly associated with alterations in the serotonergic system. Thus, the results related to elevated plus maze and force swimming test are very relevant for the study. Therefore, I recommend to include these results in the main part of the manuscript.

- For better comprehension, Figure 1 could be divided in 2 figures: behavioral data and immunohistochemistry data. Figure 2 could also be divided in 2 figures: minocycline effect and chemogenetic activation of microglia. In case all this information is maintained in Figure 2, the title should be modified.

- Figure legends should explain all abbreviations that are included in the figures.

- In Table 1 and 3 in the Supplementary Material, only the statistics of $p$ values is provided, but the obtained data for each test could be relevant to be presented.

- The affirmation Minocycline inhibits microglial activation in vivo and may have clinical utility in a variety of neurological conditions involving chronic inflammation needs references.

In the Discussion section:

-The Discussion is focused on the obtained data and hyperalgesia after adolescent alcohol exposure. The manuscript could be improved if the results about altered affective behavior are discussed as well. -It is stated that The number of 5-HT neurons was reduced in the DRN and accompanied by a reduction in 5-HT expression in the thalamus, ACC and amygdala. These regions were selected because they mediate descending modulation of pain and receive 5-HT input from the DRN. Taking into account that the manuscript includes studies related to pain sensitivity but also affective behavior, it could be relevant to mention that amygdala and thalamus are structures involved in depression and anxiety. -It is stated that a reduction in 5-HT expression in the thalamus, ACC and amygdala, but 5-HT immunoreactivity in the amygdala of ethanol-treated animals is not affected as shown in Figure 1. -The results about a decrease in the number of 5-HT+ neurons but no observed change in Tph2+ neurons appear as contradictory and should be discussed. 